published in: Bulletin of the Seismological

Society of America, vol. 91, pp. 532-537, 2001

\title{
Repeating Earthquakes as Low-Stress-Drop Events at \\ a Border between Locked and Creeping Fault Patches
}

\author{
Charles G. Sammis and James R. Rice
}

18 May 2000

Revised 11 August 2000

\begin{abstract}
The source of repeating earthquakes on creeping faults is modeled as a weak asperity at a border between much larger locked and creeping patches on the fault plane. The $x^{-1 / 2}$ decrease in stress concentration with distance $x$ from the boundary is shown to lead directly to the observed scaling $\langle T\rangle \propto\left\langle M_{0}\right\rangle^{1 / 6}$ between the average repeat time and average scalar moment for a repeating sequence. The stress drop in such small events at the border depends on the size of the large locked patch. For a circular patch of radius $R$ and representative fault parameters, $\Delta \sigma=7.6(\mathrm{~m} / \mathrm{R})^{3 / 5} \mathrm{MPa}$, which yields stress drops between 0.08 and $0.5 \mathrm{MPa}(0.8$ - 5 bars) for $R$ between $2 \mathrm{~km}$ and $100 \mathrm{~m}$. These low stress drops are consistent with estimates of stress drop for small earthquakes based on their seismic spectra. However, they are orders of magnitude smaller than stress drops calculated under the assumption that repeating sources are isolated stuck asperities on an otherwise creeping fault plane, whose seismic slips keep pace with the surrounding creep rate. Linear streaks of microearthquakes observed on creeping fault planes are trivially explained by the present model as alignments on the boundaries between locked and creeping patches.
\end{abstract}

\section{Introduction}


Seismicity on creeping sections of the San Andreas Fault has unusual temporal and spatial characteristics. Many events produce identical seismograms and, within observational limits, have the same location and the same size within 0.2 magnitude units. These events, dubbed "repeating earthquakes", have been observed at many locations in the creeping sections (Ellsworth and Dietz, 1990; Vidale et al., 1994; Marone et al., 1995; Nadeau and McEvilly, 1997; Nadeau and Johnson, 1998). Nadeau and Johnson (1998) observed that the period $T$ of repeating earthquakes scales with the scalar moment $M_{0}$ as

$$
\log \langle T / \mathrm{s}\rangle=0.17 \log \left\langle M_{0} / \text { dyne }-\mathrm{cm}\right\rangle+4.85
$$

where the brackets indicate averages over all events in a repeating sequence. This can be approximated as

$$
\langle T / \mathrm{s}\rangle=C\left(M_{0} / \text { dyne }-\mathrm{cm}\right)^{1 / 6}
$$

where we have taken $0.17 \approx 1 / 6$ and centered the fit in the middle of Nadeau and Johnson's (1998) data at $\mathrm{M}_{0}=10^{20}$ dyne-cm which gives $C=10^{4.92}$. Spatially, hypocenters in event clusters (not necessarily all repeaters) are sometimes organized into streaks, often linear streaks, up to several km long (Rubin et al.,1999).

Two questions naturally arise. First, what is the physical origin of the repeating earthquakes, i.e., why do they repeat and what determines the period? Second, why are they (and other events) organized into streaks on the fault plane? One model explored by Nadeau and Johnson (1998) and Sammis et al. (1999) is that repeating sequences occur at stuck asperities surrounded by an otherwise creeping fault plane. As pointed out by Nadeau and Johnson (1998) and reviewed in the following section, this model can lead to very large stress drops for small events that are at odds with stress drops of comparable sized events calculated from their seismic spectrum (Abercrombie, 1995; Hardebeck and 
Hauksson, 1997). While we show that it is possible to modify the assumptions in Nadeau and Johnson's analysis to yield low constant stress drop, such analyses do not simultaneously predict the observed $T \propto M_{0}^{1 / 6}$ scaling.

We develop here an alternative model for repeating earthquakes in which they nucleate in zones of weakness at the edge of much larger locked asperities in an otherwise creeping fault zone. The repeat times predicted by this model agree with the observations summarized in eqn. (2), and the stress drops are low, constant, and consistent with other spectra-based estimates for small events. This edge-crack model has the additional property that it offers a simple explanation for the observed streaks of microearthquakes; they are localized along the edges of large locked patches.

\section{Strong Asperities on a Creeping Fault Plane}

We begin with a brief review of Nadeau and Johnson's (1998) analysis in which they assume repeating earthquakes occur at isolated stuck asperities on an otherwise creeping fault plane. They used their basic observation (eqn. (1)) to find the moment release rate for each repeating sequence as

$$
\left\langle\dot{M}_{0}\right\rangle=\frac{\left\langle M_{0}\right\rangle}{\langle T\rangle}=G\langle A\rangle\langle\dot{\bar{d}}\rangle
$$

where $A$ is the area of the repeating source, $G$ is the shear modulus and $\bar{d}$ is the displacement (averaged over the slip area) of one of the repeating events. They assumed that the displacement rate on each asperity is the same (independent of $M_{0}$ ) and is equal to the displacement rate $\dot{d}_{o b s}(\approx 2.3 \mathrm{~cm} / \mathrm{yr})$ observed at the surface. Equation (3) can then be solved for the average area of the events in each sequence 


$$
\langle A\rangle=\frac{\left\langle M_{0}\right\rangle}{G\langle T\rangle \dot{d}_{o b s}} .
$$

Area was found to scale with moment as

$$
\log \left\langle A / \mathrm{cm}^{2}\right\rangle=0.83 \log \left\langle M_{0} / \text { dyne }-\mathrm{cm}\right\rangle-9.12
$$

Having estimated $\langle A\rangle$ and $\langle\bar{d}\rangle=\dot{d}_{o b s}\langle T\rangle$, Nadeau and Johnson (1998) used the elastic solution for a circular dislocation to estimate the average stress drop $\langle\Delta \sigma\rangle$ for each sequence

$$
\langle\Delta \sigma\rangle=\frac{7 \pi^{3 / 2}}{16} G \frac{\langle\bar{d}\rangle}{\langle A\rangle^{1 / 2}}
$$

which they found to scale with $M_{0}$ as

$$
\log \langle\Delta \sigma / 0.1 \mathrm{MPa}\rangle=-0.25 \log \left\langle M_{0} / \text { dyne }-\mathrm{cm}\right\rangle+8.19
$$

This result is surprising for two reasons. First, it implies that the stress-drop is higher for small events. This result is at odds with estimates based on seismic spectra that find constant stress drop consistent with established scaling relations for large earthquakes. Spectral estimates even find apparent stress drop to decrease for small events (Abercrombie, 1995; Richardson and Jordan, 2000). Second, and even more surprising, the stress levels given by (10) reach $2 \mathrm{Gpa}$ (20 kbar, more than 10 times laboratory strength) for the smallest 
events. Although Sammis et al. (1999) argued that such high stresses can not be ruled out on physical grounds, they require perfect healing (no microscopic flaws) at the smallest asperities. This is an extraordinary result that demands a close scrutiny of the assumptions in the Nadeau and Johnson (1998) analysis.

Two points in the Nadeau and Johnson (1998) analysis can be questioned: a) the use of the crack equation (9) to analyze stress on an asperity, and b) the basic assumption that the displacement rate on individual asperities is equal to that observed at the surface.

Beginning with point (a), Das and Kostrov (1986) analyzed the fracture mechanics of the annular crack $a<r<b$ shown in Fig. 1a. The region $r<a$ is the asperity and the region $a<r<b$ is the stress-free creeping region. They found

$$
\frac{\bar{\sigma}_{a}}{\sigma_{\text {remote }}}=0.8 \frac{b}{a}
$$

where $\bar{\sigma}_{a}$ is the average shear stress on the asperity when the remote stress is $\sigma_{\text {remote }}$. The stress intensity factor for the asperity (at $r=a$ ) is, for $b>>a$, (Tada et al. 1983)

$$
K \approx \frac{\sigma_{\text {remote }}}{\sqrt{a}} b\left(\frac{4}{\pi^{3 / 2}}\right)
$$

The asperity fails when $K$ equals the critical stress intensity factor $K_{C}$ (a material property) and eqn. (9) may be combined with eqn. (8) to yield the failure stress $\bar{\sigma}_{a}{ }^{*}$

$$
\bar{\sigma}_{a}^{*}=1.1 \frac{K_{c}}{\sqrt{a}}
$$


where the outer radius of the annular crack $b$ has dropped out of the equation. The average displacement on the asperity when it fails, $\langle\bar{d}\rangle^{*}$, is approximately that at the center of a stress-free crack of radius b in a stress field $\sigma_{\text {remote }}$ which, from equations (6) and (9) is

$$
\langle\bar{d}\rangle^{*}=\frac{4 \sqrt{\pi}}{7} \frac{K_{c}}{G} \sqrt{a}
$$

Note that $\bar{\sigma}_{a}^{*} \propto\langle\bar{d}\rangle^{*} / a$ as required.

Equations (10) and (11) show that the scaling behavior of the stress-drop and displacement on a hard asperity depends on the scaling properties of the critical stress intensity factor in shear ( $K_{I I c}$ and/or $K_{I I I c}$ depending on loading). If $K_{I I c}$ and $K_{I I I c}$ are scale independent constants (as is generally assumed for $K_{I c}$ in tensile loading) then the stress-drop scales as $a^{-1 / 2}$ and eqn. (10) shows that small events are expected to have larger stress drops. For $K_{c}=$ constant, eqn. (11) gives $\langle\bar{d}\rangle \propto \sqrt{a}$ which, for constant loading rate, leads to $T \propto\langle\bar{d}\rangle \propto \sqrt{a} \propto M_{0}{ }^{1 / 6}$ as observed. However, shear failure under compressive loading is a complex process involving the nucleation, growth, and interaction of a myriad of smaller tensile fractures in a process zone near the crack tip (Ashby and Sammis, 1990). Scholz et al. (1993) argue that the energy release rate for shear propagation, $\mathcal{G}_{I I}$ or $\mathcal{G}_{I I I}$, increases linearly with fracture dimension $a$. Since $\mathcal{G}$ is related to the stress intensity factor as

$$
\mathcal{G}_{c} \propto \frac{K_{c}^{2}}{E} \propto a
$$

where $E$ is the appropriate elastic modulus for the loading mode, it follows that $K_{c} \propto a^{1 / 2}$. In this case, equations (10) and (11) give constant stress drop and displacement 
proportional to $a$. However, $\langle\bar{d}\rangle \propto a$ implies $T \propto M_{0}{ }^{1 / 3}$ contrary to observation. Hence, given the observation $T \propto M_{0}{ }^{1 / 6}$, there is no fundamental difference between the scaling properties of the crack and the asperity model The use of an asperity model in place of the crack model used by Nadeau and Johnson (1998) does not solve the high stress drop problem.

The situation may be summarized by the following 3 simple statements: 1) constant stress drop requires $d \propto M_{0}^{1 / 3}$ for either the crack or the asperity model, 2) If the displacement-rate on all asperities is the same (ie. $\dot{d}=d\left(M_{0}\right) / T\left(M_{0}\right)=$ constant), then $T \propto M_{0}{ }^{1 / 3}$, and 3) Nadeau and Johnson (1998) observe a much weaker dependence of T on $\mathrm{M}_{0}$ more like $T \propto M_{0}{ }^{1 / 6}$. If we accept the observed dependence of repeat time on moment, then there are only two possibilities: either 1) is false and the stress drop is not constant (Naduau and Johnson's 1998 conclusion), or 2) is false and the seismic displacement-rate associated with the repeating events depends on moment. However, the total displacement rate must be the same on all asperities over time to assure continuity of slip on the fault surface. Hence the second possibiltiy can be restated; the seismic displacement-rate associated with repeating events on an asperity must be less that its total long-term displacement rate. There are at least two ways for this to happen: 1) a smaller asperity experience significant displacement during the rupture of a larger neighboring asperity, or 2) an asperity experience significant aseismic creep between repeating events.

The first possibility was suggested by Anooshehpoor and Brune (1998). They used a foam rubber fault simulator (Brune et al., 1993; Anooshehpoor and Brune, 1994) to explore the geometry in Fig. 1. The stress-free annulus in the region $a<r<b$ was produced by inserting a smooth plastic semi-annulus annulus between the foam blocks as indicated in Fig. 1b. The asperity was observed to fail many times for each slip event on the larger fault plane. In this experiment the observed surface displacement rate is dominated by the larger 
system-wide events. To assign this surface rate to the smaller events on the asperity leads to a gross overestimate of their stress drop. One could imagine a generalization of this model in which a hierarchy of larger asperities shields smaller ones. The key characteristic of this model is that the asperities are not isolated in the sense that larger events produce slip on smaller asperities. Hence, a repeating sequence only represents a small fraction of the total displacement on an asperity over time.

The shielded asperity model can give a low constant stress drop, but can it also produce the observed $T \propto M_{0}{ }^{1 / 6}$ scaling of repeat time? If $T \propto M_{0}{ }^{1 / 6}$, and the stress drop is constant $\left(d \propto a \propto M_{0}{ }^{1 / 3}\right)$, then $T \propto \sqrt{d}$, or equivalently, $T \propto \sqrt{a}$. The only way to get this dependence in a constant stress-drop model is for the loading rate on an asperity to scale as $\dot{\sigma} \propto a^{-1 / 2}$. From equation (10), the loading rate on an asperity is $\dot{\sigma}=0.8(b / a) \dot{\sigma}_{\text {remote }}$. For constant remote stressing rate, $\dot{\sigma} \propto a^{-1 / 2}$ requires $b \propto \sqrt{a}$. While it is thus possible for the hard asperity model to satisfy the observed repeat time scaling with constant stress drop, we can think of no reason for the clustering geometry implied by $b \propto \sqrt{a}$. We will show in the next section that this requisite scaling of the loading rate occurs naturally for soft asperities at the edge of a much larger hard asperities.

The second possibility, where significant aseismic deformation occurs between repeating events, was proposed by Beeler (2000) who developed a strain-hardening model. His model is motivated by laboratory experiments on serpentinite gouge in which significant aseismic creep precedes each stick-slip event in a repeating sequence. Beeler captures this behavior with a simple analog spring slider system having a critical stress threshold for slip and a linear displacement-hardening slip function. When this hardening is small, the fault creeps at some creep strength $\tau_{0}$, well below the critical slip stress $\tau_{s}$. When the hardening coefficient is large, very little aseismic creep occurs and the system behaves like a simple relaxation oscillator cycling between $\tau_{0}$ and $\tau_{s}$. When the loading stiffness 
and hardening coefficient are comparable, stick slip oscillations are preceded by significant aseismic creep - as in the experiments. By thus allowing the displacement associated with the repeating events to be well below the surface slip rate, this model also allows for reasonable stress drops. In Beeler's model the weak dependence observed between repeat time and seismic moment is associated with a transition from aseismic creep to stick slip behavior. However, this is a progressive transition which does not give $T \propto M_{0}{ }^{1 / 6}$ scaling over a very wide range of moments.

In the next section we develop a model similar to that studied by Anooshehpoor and Brune (1998), but without the hard asperity. We will show that a weak spot at the edge of a generally stronger large asperity can produce a realistic model for repeating earthquakes with low stress drops and $T \propto M_{0}^{1 / 6}$ scaling.

\section{Weak Asperities at the Border between Locked and Creeping Fault Patches}

The challenge, as discussed above, is to find a model for the repeating earthquakes in which the loading rate on the asperities scales as $\dot{\sigma} \propto a^{-1 / 2}$. In that case, a constant stress-drop model will have the observed $T \propto M_{0}{ }^{1 / 6}$ scaling. This $\dot{\sigma} \propto a^{-1 / 2}$ scaling is precisely what would be expected if the repeating events are failures of small weak patches located at the edges of larger, and generally stronger, asperities as illustrated in Fig. 2. The stress field at the boundary of a large locked asperity is proportional to $x^{-1 / 2}$ where $x$ is the distance from the boundary. Hence the average stress across a weak spot of radius $a$ located at the boundary (as in Fig. 1a) is proportional to $a^{-1 / 2}$, and hence the repeat time will be proportional to $\sqrt{a}$ as observed. 
We can take this analysis one step further and estimate the stressing rate $\dot{\sigma}$ for the case where the large asperity is circular as illustrated in Fig. 2b. In this case the stress distribution on the large asperity of radius $\mathrm{R}$ is (Das and Kostrov, 1986)

$$
\sigma(\rho)=\frac{8}{7 \pi} \frac{G \bar{d}}{\sqrt{R^{2}-\rho^{2}}}
$$

where $0 \leq \rho \leq R$. If $a$ is the radius of a small weak patch at the border and $0 \leq \alpha \leq a$ as in

Fig. 1b, then for $\alpha<<R, \sqrt{R^{2}-\rho^{2}} \approx \sqrt{2 R \alpha}$ and eqn. (13) may be written

$$
\sigma(\alpha)=\frac{8}{7 \pi} \frac{G \bar{d}}{\sqrt{2 R}} \frac{1}{\sqrt{\alpha}}
$$

The average stressing rate on the small patch, with $a<<R$, is then approximately

$$
\dot{\bar{\sigma}} \approx \frac{1}{2 a} \int_{0}^{a} \dot{\sigma}(\alpha) d \alpha=\frac{8}{7 \pi} \frac{G \dot{\bar{d}}}{\sqrt{2 R}} \frac{1}{\sqrt{a}}
$$

Here $\dot{\bar{d}}$ is the average displacement rate on the large asperity which is assumed to equal the observed rate over the long term. We can also write

$$
\dot{\sigma}=\frac{\langle\Delta \sigma\rangle}{\langle\Delta T\rangle}=\frac{\langle\Delta \sigma\rangle}{C\left(M_{0} / \text { dyne }-\mathrm{cm}\right)^{1 / 6} \mathrm{~s}}=\frac{\langle\Delta \sigma\rangle^{5 / 6}}{C\left(\frac{16}{7 \text { dyne }-\mathrm{cm}}\right)^{1 / 6} a^{1 / 2} \mathrm{~s}}
$$


where we have used eqn. (6) for the stress drop on a circular dislocation of radius $a=\sqrt{A / \pi}$ and the defintion of moment to write $\Delta \sigma=7 M_{0} / 16 a^{3}$. Equating (15) and (16) gives a relation between the stress drop to the size of the large asperity $\mathrm{R}$

$$
\Delta \sigma=\left(\frac{16}{7}\right)^{7 / 5}\left(\frac{C G \dot{\bar{d}}}{2 \pi \sqrt{2 R}}\right)^{6 / 5}\left(\frac{\mathrm{s}^{6}}{\text { dyne }-\mathrm{cm}}\right)^{1 / 5}
$$

We observe that the stress drop predicted by this model is independent of the size $a$ of a small region failing at the border of the larger locked patch. Hence, for a given large locked asperity size $R$, the stress drop is constant, independent of the small event size.

Substituting in $G=30 \mathrm{GPa}=3 \times 10^{10} \mathrm{Nm}^{-2}, \dot{\bar{d}}=2.3 \mathrm{~cm} / \mathrm{yr}=7.3 \times 10^{-10} \mathrm{~m} / \mathrm{s}$, and $C=10^{4.92}$ gives that stress drop as

$$
\Delta \sigma=7.6(m / R)^{3 / 5} M P a
$$

For large asperities with radii in the range $100 \mathrm{~m}-2 \mathrm{~km}$ the stress drop ranges from about $0.5 \mathrm{MPa}$ to $0.08 \mathrm{MPa}$ (5 to $0.8 \mathrm{bars}$ ). It is interesting that this is at the lower limit of the range of stress drops found by Abercrombie (1995) using spectral corner frequency methods for small earthquakes in Southern California. On the other hand, quite low stress drops are consistent with repeated ruptures of weak edge regions before the rest of the "strong" asperity fails in a larger event. The dependence of stress drop on the size of the larger locked asperity may explain some of the scatter in these data which typically exceeds an order of magnitude in stress drop for a given seismic moment. 


\section{Discussion}

The stress drop calculated for repeating microearthquakes is very sensitive to the assumed source model. Starting with the same observed scaling of repeat time with moment, as in equation (2), the isolated stuck asperity model yields stress drops on the order of $2 \mathrm{GPa}$ (20 kbar) while the weak border crack model yields $0.1-1.0 \mathrm{MPa}$ (1-10 bars). The former assumes that each asperity tracks the surface displacement rate, which means that displacement of each event is proportional to repeat time. Substituting $\langle d\rangle \propto\langle T\rangle$ into the basic observation $\langle T\rangle \propto M_{0}^{1 / 6}$ yields $d \propto A^{1 / 4}$. Since constant stress drop requires $d \propto A^{1 / 2}$, the isolated stuck asperity model yields a stress drop dependent on moment and leads to very high stress drops for small events. The weak border crack model, on the other hand, implies uniform stress drop at a given border and hence $d \propto A^{1 / 2}$ which, when substituted into the observed $\langle T\rangle \propto M_{0}{ }^{1 / 6}$, yields $\langle T\rangle \propto \sqrt{d \propto} \sqrt{a}$ where $a$ is the

source radius. This model uses the $x^{-1 / 2}$ stress concentration at the edge of a larger hard asperity to achieve the required scaling of $T$ with $a$. The low stress drops in this latter model are a consequence of the fact that the short-term displacement-rate on the weak asperities does not equal the displacement-rate observed at the surface. The vast majority of displacement on these edge asperities occurs when the larger strong asperity fails, just as in Anooshehpoor and Brune's (1998) related (but different) foam rubber model. This conclusion is not at odds with recent evidence from Nadeau and McEvilly (1999) that changes in $T$ correlate with changes in the surface creep rate. The asperities can track the surface rate without being equal to it. The two rates are equal in Nadeau and McEvilly's analysis precisely because that is the basic assumption in Nadeau and Johnson (1998).

The border, or edge, crack model is supported by several observations. First, seismicity at Parkfield is limited to the depth range between 2 and $11 \mathrm{~km}$ with rather linear 
upper and lower boundaries (Fig. 2) Modeling of geodetic surface displacements by Tse et al. (1985) suggest that these may be the upper and lower bounds of the locked asperity. Repeating earthquakes tend to cluster near these boundaries. Finally, hypocenters of small earthquakes in the creeping sections of the San Andreas fault tend to form horizontal lines. In their discussion of these observations, Rubin et al. (1999) raised the question of whether the quiet areas between streaks have no earthquakes because they are creeping or because they are locked. They logically concluded that they are probably creeping since creep is required to reload asperities to produce repeating sequences and the rates of repeating earthquakes were observed to increase in response to the Loma Prieta earthquake. We raise the possibility here that some quiet patches are creeping and some are locked with repeating earthquakes occurring at the boundary between them. 


\section{Acknowledgments}

We wish to thank Greg Beroza for suggesting a focus on borders between locked and creeping zones, and Jim Brune, Bob Nadeau, and Allan Rubin for helpful discussions about repeating earthquakes.. This work was supported by NSF grants NSF-EAR-9902901 (CGS) and EAR-9805182 (JRR).

\section{References}

Abercrombie, R. E.(1995). Earthquake source scaling relationships from -1 to 5 ML using seismograms recorded at 2.5-km depth, J. Geophys. Res. 100, 24,015 - 24,036.

Anooshehpoor, A., and J. N. Brune (1994). Frictional heat generation and seismic radiation in a foam rubber model of earthquakes, Pure Appl. Geophys 142, 735-747.

Anooshehpoor, A. and J. N. Brune (1998). Quasi-static slip-rate shielding by locked and creeping zones as an explanation of small repeating earthquakes at Parkfield (abstract), EOS Trans. Am. Geophys. Union. 79, F594.

Ashby, M.F. and C.G. Sammis (1990). The damage mechanics of brittle solids in compression, Pure and Appl. Geophys.,133, 489-521.

Beeler, N.M.(2000). A simple stick-slip and creep-slip model for repeating earthquakes and its implication for micro-earthquakes at Parkfield, Bull. Seism. Soc. Am., submitted.

Brune, J. N., S. Brown, and P. A. Johnson (1993). Rupture mechanism and interface separation in foam rubber models of earthquakes: a possible solution to the heat flow paradox and the paradox of large overthrusts, Tectonophysics 218, 59-67. 
Das, S. and B. V. Kostrov (1986). Fracture of a single asperity on a finite fault: A model for weak earthquakes?, in Earthquake Source Mechanics,. S. Das, J. Boatwright, and C.H. Scholz (Editors), Am. Geophys. Union, M. Ewing Vol. 6, Geophys. Monogr.37, 91-96.

Ellsworth, W. L., and L. D. Dietz (1990). Repeating earthquakes: characteristics and implications, Proc. of Workshop, XLVI, the 7th U.S. - Japan Seminar on Earthquake Prediction, U.S. Geol. Surv. Open-file Rept. 90-98, 226-245.

Hardebeck, J. L., and E. Hauksson (1997). Static stress drop in the 1994 Northridge, California, aftershock sequence, Bull. Seism. Soc. Am. 87, 1495-1501.

Marone, C., J. E. Vidale, and W. Ellsworth (1995). Fault healing inferred from time dependent variations in source properties of repeating earthquake, Geophys. Res. Lett. 22, 3095-3098.

Nadeau, R. M. and L. R. Johnson (1998). Seismological studies at Parkfield VI: Moment release rates and estimates of source parameters for small repeating earthquakes, Bull. Seism. Soc. Am. 88, 790-814.

Nadeau, R. M. and T. V. McEvilly (1999). Fault slip rates at depth from recurrence intervals of repeating microearthquakes, Science, 285, 718-721.

Nadeau, R. M. and T. V. McEvilly (1997). Seismological studies at Parkfield V: Characteristic microearthquake sequences as fault-zone drilling targets, Bull. Seism. Soc. Am. 87, 1463-1472.

Richardson, E., and T.H. Jordan (2000). Seismicity in deep gold mines of South Africa: Implications for tectonic earthquakes, Bull. Seism. Soc. Am. submitted.

Rubin, A. M., D. Gillard, and J.-L. Got, Streaks of microearthquakes along creeping faults, Nature, 400, 635-641, 1999.

Sammis, C. G., R. M. Nadeau, and L. R. Johnson (1999). How Strong is an asperity?, J. Geophys. Res. 104, 10,609-10,619. 
Scholz, C.H., N.H. Dawers, J.-Z. Yu, M.H. Anders, and P.A Cowie, Fault growth and fault scaling laws: Preliminary results, J. Geophys. Res., 98, 21,951-21,961, 1993.

Tada, H., Paris, P. C. and Irwin, G. R.(1985). The Stress Analysis of Cracks Handbook, Paris Productions, Inc., Saint Louis, MO.

Tse, S. T., R. Dmowska, and J. R. Rice (1985). Stressing of locked patches along a creeping fault, Bull. Seism. Soc. Am. 75, 709-736.

Vidale, J. E., W. Ellsworth, A. Cole, and C. Marone (1994). Rupture variation with recurrence interval in eighteen cycles of a small earthquake, Nature 368, 624-626.

Department of Earth Sciences

University of Southern California

Los Angeles, CA 90089-0740

email: sammis@earth.usc.edu

(C.G.S.)

Department of Earth and Planetary Sciences

and Division of Engineering and Applied Sciences

Harvard University

Cambridge, MA 02138

email: rice@esag.harvard.edu.

(J.R.R) 


\section{Figure Captions}

Figure 1. The strong asperity model. a) shows the asperity of radius $a$ surrounded by the stress free annulus of radius $b$. b) is a sketch of the foam rubber block experiment used by Anooshehpoor and Brune (1998) to simulate repeating earthquakes.

Figure 2. The weak border asperity model. a) shows a weak asperity of radius $a$ at the boundary between a locked and creeping patch of the fault plane. The stress concentration in the locked portion decreases as $x^{-1 / 2}$ with distance $x$ from the boundary. b) shows a weak circular asperity at the boundary of a larger circular locked patch. The radius of the locked patch is $\rho$ where $0<\rho<R$ while the radius of the weak asperity is $\alpha$ where $0<\alpha<a$. 

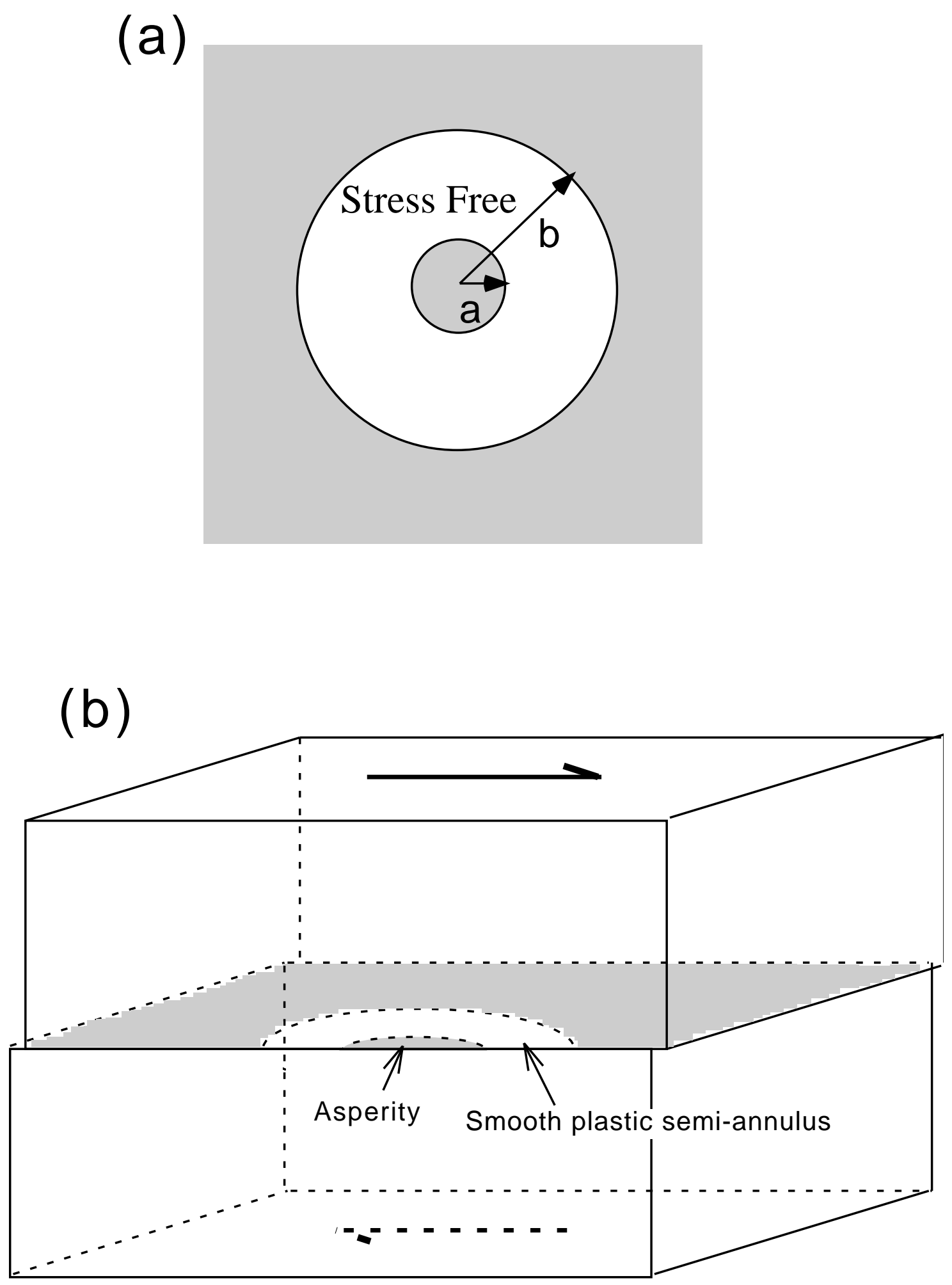
(a)

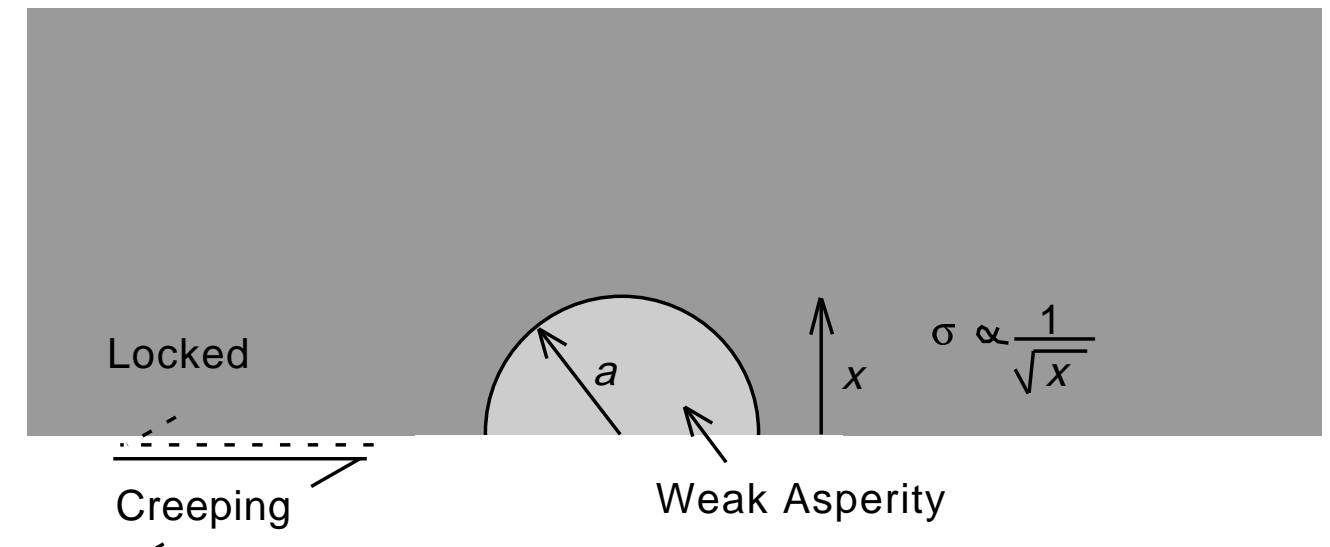

(b)

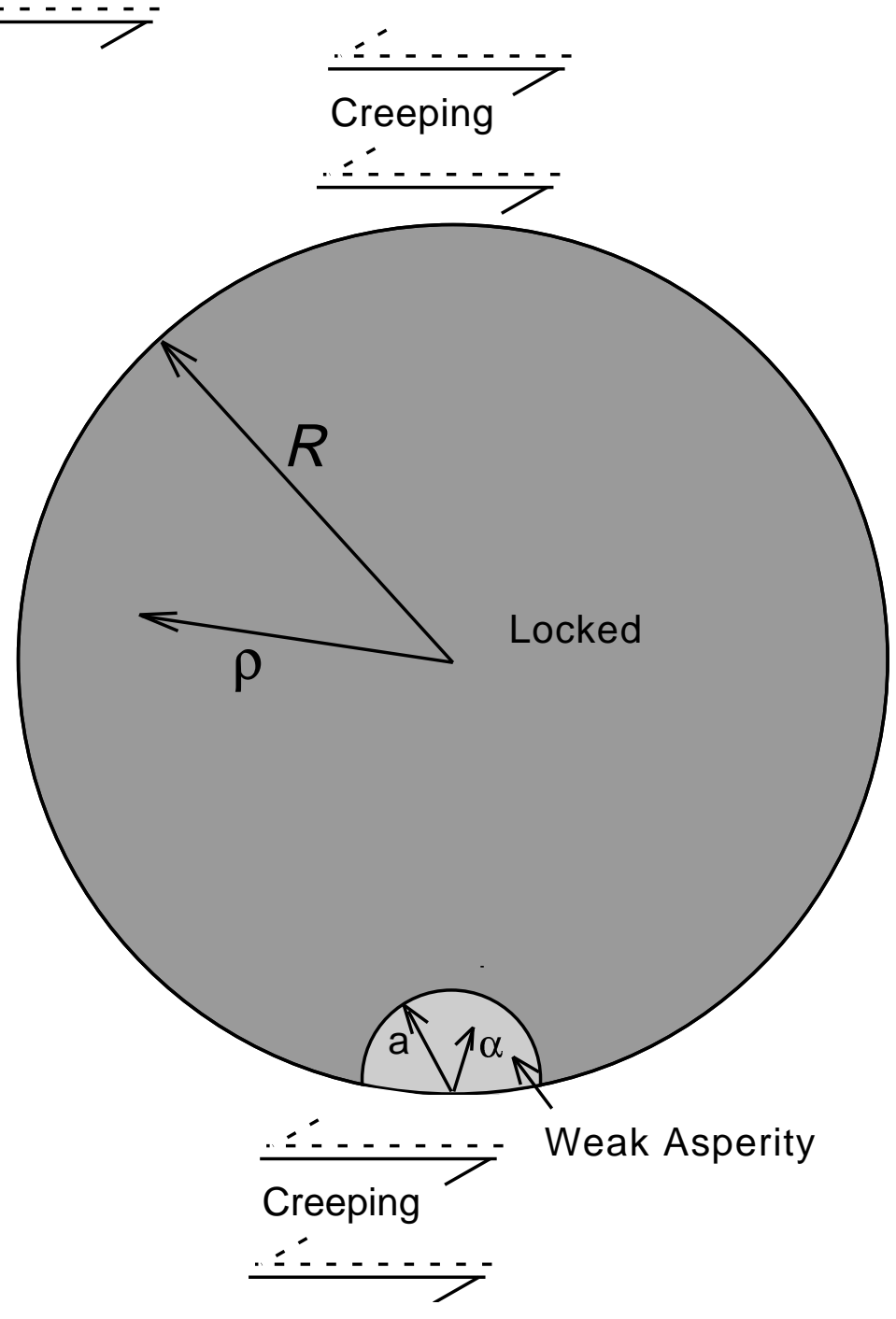

